\title{
BRAIDED ENDOMORPHISMS OF CUNTZ ALGEBRAS
}

\author{
ROBERTO CONTI and FRANCESCO FIDALEO
}

\begin{abstract}
We discuss sufficient conditions ensuring that certain endomorphisms of infinite factors arising from Cuntz algebras are braided. We analyze some explicit non-trivial examples associated to unitary solutions of the quantum Yang-Baxter equation on a Hilbert space of dimension 2. In particular we show the existence of endomorphisms of index 2 associated to representations of Hecke algebras at a primitive fourth root of unity. In this case we compute the associated fusion rules. These fusion rules define a finitely generated ${ }^{*}$-semiring which is not finite. Such a picture seems to be closely related to the description of (the dual of) a deformation, at a fourth root of unity, of some compact matrix group. This could be of some interest for the investigation of quantum symmetries naturally appearing in low-dimensional Quantum Field Theory.
\end{abstract}

\section{Introduction}

In the last years the interest in the study of inclusions of von Neumann algebras (up to coniugacy) and their main invariants as the index and the paragroup had a considerable growth [32,33]. On the other hand, endomorphic inclusions of algebras of localized observables make their appearence also in Quantum Field Theory (QFT) since they describe the superselection structure of a given theory $[6,11]$. Inclusions arising from endomorphisms of a factor were considered in [26], and very remarkable relations were discovered between the theory of invariants of a given inclusion and the theory of the (statistical) dimension of (localized) endomorphisms appearing in QFT, see [25, 26, 27]. More recently these connections were also studied in the more general framework of tensor $C^{*}$-categories [29].

A relevant fact is that representations of Artin braid group naturally appear in QFT due to the indistinguishibility of quantum particles, and therefore link invariants $([13,42])$ are canonically attached to any sector $[26,27]$ as in the case of a given inclusion of von Neumann algebras [20]. For theories living in the physical Minkowski space-time, the representations of the braid group degenerate in representations of the permutation group [6] and

\footnotetext{
* Partially supported by CNR-GNAFA.

Received August 26, 1997.
} 
the emerging structure is completely clarified [7]. The statistical dimension of a sector, which coincides with the square root of the index of suitable inclusions of local algebras [25], completely classifies the classes of the representations of the permutation group relative to that sector and it is a relevant input in order to recover the observable algebra via a principle of gauge invariance [7]. However in the case of theories living in a low-dimensional space-time manifold the corresponding gauge structure is not yet fully understood and it seems to be much more complicated [34, 36].

Motivated by these facts the structure of braided endomorphism, and in particular those which are standard, are intensively studied in [27, 35]. For low-channel sectors, the associated representations of the braid group are classified in terms of representations of Hecke or Birman-Wenzl-Murakami algebras, see also [11]. Unfortunately, all the known examples of (standard) braided endomorphisms are very complicated [30, 38] so it is certainly worthwhile constructing and studying other explicit cases. On the other hand unital endomorphisms of a Cuntz algebra arise in a very simple way [5], and one could argue that, if braided ones exist, then they have to be necessarily simpler.

In a Cuntz algebra $\mathcal{O}_{d}$, a unitary $U \in \mathcal{O}_{d}$ defines an endomorphism $\lambda_{U} \in \operatorname{End}\left(\mathcal{O}_{d}\right)$ via its action on the generating Hilbert space $H \subset \mathcal{O}_{d}$

$$
T \in H \rightarrow \lambda_{U}(T):=U T,
$$

and every (unital) endomorphism arises in this way [5]. Interesting classes of endomorphisms are generated by unitaries $V=R F$ with $R \in\left(H^{2}, H^{2}\right)$ satisfying the pentagon equation [5] ( $F$ is the unitary implementing the flip operator in $H^{2}$, see below). These endomorphisms are closely related to actions of finite dimensional Kac algebras on von Neumann factors [28]. Other relevant classes of endomorphisms are associated with unitaries $Y=R F$ where $R \in\left(H^{2}, H^{2}\right)$ satisfies the Yang-Baxter equation without spectral parameter [37].

Some properties of the indices associated with certain endomorphisms of the Cuntz algebras (or of their UHF subalgebras as well) were first investigated in [17, 22, 28]. Later, they were sistematically studied in [3], where many considerations about the Yang-Baxter endomorphisms were also made.

The Yang-Baxter endomorphisms seem to be the natural candidates to give examples of standard braided endomorphisms. In fact, if $R=Y F$ satisfies the Yang-Baxter condition, then $Y \in\left(H^{2}, H^{2}\right)$ satisfies the equation

$$
Y \lambda_{Y}(Y) Y=\lambda_{Y}(Y) Y \lambda_{Y}(Y)
$$

therefore 


$$
\pi\left(\sigma_{i}\right):=\lambda_{Y}^{i-1}(Y), \quad i=1,2, \ldots
$$

defines a unitary representation of the braid group $\mathrm{B}_{\infty}$ inside the Cuntz algebra. In the irreducible case a link invariant is also defined via a canonical Markov trace $[42,27]$. If the endomorphism $\lambda_{Y}$ is standard braided w.r.t. $Y$ (i.e. $\lambda_{Y}$ is $Y$-braided for short), that is

$$
d\left(\lambda_{Y}\right)=\left|\Phi_{\lambda_{Y}}(Y)\right|^{-1},
$$

then the structure of the tower of relative commutants $\left(\lambda_{Y}^{n}, \lambda_{Y}^{n}\right)$ seems closely related to that of the inclusion $\lambda_{Y}(M) \subset M$ ([29]) where $M$ is a canonical type-III factor related to $\mathcal{O}_{d}$, see below.

A similar situation appears in QFT, where the analog of $\cup_{n}\left(\lambda_{Y}^{n}, \lambda_{Y}^{n}\right)$ plays a central role in the description of the observable algebra via a principle of gauge invariance $[7,34,36]$. This has been succesfully exploited in the case of theories living on Minkowski space-time, where a compact gauge group is involved [7].

The aim of this paper is to study conditions for a Yang-Baxter endomorphism $\lambda_{Y}$ to be $Y$-braided, that is the statistical operator of $\lambda_{Y}$ (in the language of QFT [6, 11]) is exactly $Y$. In general this is not automatic. However, when our conditions are fulfilled, we can exhibit very explicit examples of braided endomorphisms. Namely, we show that many nontrivial examples of standard braided endomorphisms of the Cuntz algebra $\mathcal{O}_{2}$ exist, and in particular with index 2, a problem left open in [3].

The final part of this paper is devoted to the study of the fusion rules generated by the above (essentially unique) Yang-Baxter endomorphism of $\mathrm{O}_{2}$ of index 2. Hopefully, the detailed knowledge of such fusion rules could be strictly related to the description of quantum symmetries like those arising in low-dimensional QFT [31]. The action of this (hidden) symmetry on a factor (or equally well on a field algebra in QFT) has to be recovered looking at its regular representation viewed as a Longo's canonical endomorphism $[10,28]$.

\section{Preliminaries}

For each integer $d \geq 2$ we denote by $\mathcal{O}_{d}$ the Cuntz algebra, that is the $C^{*}$ algebra generated by a Hilbert space $H$ spanned by $d$ isometries $T_{i}, i=1, \ldots, d$ with mutually orthogonal ranges summing up to the identity [4]. As usual, we denote by $\left(H^{r}, H^{s}\right)$ the image of the natural embedding of $\operatorname{Hom}\left(H^{\otimes r}, H^{\otimes s}\right)$ inside $\mathcal{O}_{d}$. Then $\mathcal{O}_{d}$ has a natural inductive limit structure with the inclusion $\left(H^{r}, H^{s}\right) \subset\left(H^{r+1}, H^{s+1}\right)$ corresponding to tensorization on the right by the identity operator. 
Any unitary $U \in \mathcal{O}_{d}$ gives rise to an endomorphism $\lambda_{U}$ of $\mathcal{O}_{d}$ completely determined by

$$
\lambda_{U}(T)=U T, \quad T \in H
$$

and any unital endomorphism arise in this way [5]. The inner endomorphism

$$
\varphi_{H}: X \rightarrow \sum_{i=1}^{d} T_{i} X T_{i}^{*}
$$

is induced by the self-adjoint unitary flip $F: T S \rightarrow S T, S, T \in H$ and it is independent on the chosen orthonormal basis. In the sequel we often drop the subscript $H$ when there is no matter of confusion.

A Yang-Baxter endomorphism (YB for short) is an endomorphism of the Cuntz algebra associated with a unitary $Y=R F$, with $R \in\left(H^{2}, H^{2}\right)$ satisfying the YB equation (without spectral parameter) $[15,37]$. The YB equation translates, in the Cuntz algebra language, in the following condition for $Y$ [5]:

$$
Y \varphi(Y) Y^{\sharp}=\varphi\left(Y^{\sharp}\right) Y \varphi(Y)
$$

where, for generic $X \in \mathcal{O}_{d}$, we write $X^{\sharp}$ for $X$ or $X^{*}$ indifferently. The above equation is also equivalent to

$$
\lambda_{Y}(Y)=\varphi(Y) .
$$

We denote by $\mathrm{YB}\left(\mathcal{O}_{d}\right)$ the set of unitaries satisfying the $\mathrm{YB}$ equation as above. Well-known elements in $\mathrm{YB}\left(\mathcal{O}_{d}\right)$ are $z I, z \in \mathrm{C},|z|=1$, and $F$.

The Cuntz algebra $\mathcal{O}_{d}$ is endowed with a canonical Z-grading associated to the automorphic action of the torus $z \in \mathrm{T} \rightarrow \alpha_{z}:=\lambda_{z I}$. The fixed point algebra $\mathcal{O}_{d}^{(0)}$ coincides with $\left(\bigcup_{n} \mathscr{M}_{n}\right)^{-\|\cdot\|}$, where $\mathscr{M}_{r}:=\left(H^{r}, H^{r}\right)$ is isomorphic to the algebra of $d^{r} \times d^{r}$ complex matrices, and hence it is the UHF algebra of type $d^{\infty}$ equipped with a canonical faithful trace $\tau$. In particular $\varphi \mid \mathcal{O}_{d}^{(0)}$ can be identified with the one-step unilateral shift on the right. Denoting by $m$ the faithful conditional expectation $\mathcal{O}_{d} \rightarrow \mathcal{O}_{d}^{(0)}$ associated with the T-action, $\omega:=\tau \circ m$ is a faithful state on $\mathcal{O}_{d}$. The weak closure $M$ of $\mathcal{O}_{d}$ in the GNS representation $\pi_{\omega}$ of $\omega$ is a copy of the unique AFD factor of type III $_{\frac{1}{d}}$. Any $\lambda_{U}$ with $U \in\left(H^{r}, H^{r}\right)$ can be uniquely extended by $\omega$-invariance to a (normal) endomorphism of $M$ (which will still be denoted in the sequel as $\lambda_{U}$ ) with finite Jones-Kosaki-Longo index [28]. The action $\alpha$ can be extended to an action on $M$, and (the normal extension of) $\omega$ is a KMS state with respect to the 1-parameter (periodic) modular automorphism group $\sigma_{t}^{\omega}=\alpha_{d^{-i t}}$, $t \in \mathrm{R}$. The centralizer $M^{(0)}:=M^{\sigma^{\omega}}$ is a copy of the AFD factor of type $\mathrm{II}_{1}$ 
$[17,28]$. Furthermore $([3,28])$ we have the following upper bound for the index:

$$
\operatorname{Ind}\left(E_{U}\right)=\left[M^{(0)}: \lambda_{U}\left(M^{(0)}\right)\right] \leq d^{2(r-1)}
$$

where $E_{U}$ is the unique $\omega$-invariant conditional expectation $M \rightarrow \lambda_{U}(M)$ and $[:]$ is the Jones index, see also below.

In the following, given any properly infinite von Neumann algebra $M$, $\operatorname{End}(M)$ will denote the semigroup of all unital injective normal *-endomorphisms of $M, \operatorname{Aut}(M) \subset \operatorname{End}(M)$ the group of automorphisms and $\operatorname{Inn}(M)$ the normal subgroup of inner automorphisms. We refer the reader to $[19,24,33]$ for the general theory of the index for inclusions of von Neumann algebras and to $[16,25,26,27]$ for sector theory.

Given $\rho, \sigma \in \operatorname{End}(M)$, the space of intertwiners between $\rho$ and $\sigma$ is

$$
(\rho, \sigma):=\{v \in M \mid v \rho(x)=\sigma(x) v, x \in M\} .
$$

Given an inclusion $N \subset M$ of properly infinite von Neumann algebras on a separable Hilbert space, there are joint cyclic and separating vectors $\xi$. The canonical endomorphism $\gamma:=\operatorname{ad}\left(J_{N} J_{M}\right): M \rightarrow N$, with $J_{M}$ (resp. $\left.J_{N}\right)$ the modular conjugation of $M$ (resp. $N$ ) w.r.t. some fixed common standard vector $\xi$ is unique up to inner automorphisms of $N$. Conversely any canonical endomorphism is of the form $\gamma=\rho \bar{\rho}$ provided $N=\rho(M)$ is isomorphic to $M$ (see below).

If $\rho \in \operatorname{End}(M)$, a conjugate is provided by $\bar{\rho}=\rho^{-1} \circ \gamma_{\rho}$, with $\gamma_{\rho}: M \rightarrow$ $\rho(M)$ a canonical endomorphism; then $\bar{\rho}$ is uniquely defined up to inner automorphisms and thus there is a well-defined conjugation in the semiring $\operatorname{Sect}(M):=\operatorname{End}(M) / \operatorname{Inn}(M)[26]$.

Given $\rho \in \operatorname{End}(M)$, let $E: M \rightarrow \rho(M)$ be a conditional expectation of $M$ onto $\rho(M)$; then we can associate a left inverse of $\rho$ by $\Phi:=\rho^{-1} \circ E$. It turns out that this correspondence is a bijection with inverse $E=\rho \Phi$.

Given any normal faithful conditional expectation $E: M \rightarrow N$, it is uniquely defined an operator-valued weight $E^{-1}: N^{\prime} \rightarrow M^{\prime}$ such that $E^{-1}(I) \in \overline{Z(M)_{+}}$, the extended positive part of $M$. The expectation $E$ is said to be of finite index if $E^{-1}(I) \in Z(M)$, and in this case $\operatorname{Ind}(E):=E^{-1}(I)$ is called the index of $E$ [24]. In the case of a factor-subfactor inclusion it is well known that if there exists a conditional expectation of finite index (i.e. $\operatorname{Ind}(E) \in \mathrm{R}_{+}$), then there exists exactly one for which the index attains its minimal value $[14,25]$. In the more general case of an inclusion of von Neumann algebras the situation is quite different [9].

We say that $\rho$ has finite index if there exists $E: M \rightarrow \rho(M)$ of finite index. 
We denote by $\operatorname{End}_{0}(M)$ the $*$-subring of endomorphisms with finite index, and by $\operatorname{Sect}_{0}(M)$ the corresponding quotient modulo inners.

Given an infinite factor $M$ and $\rho \in \operatorname{End}_{0}(M)$ we denote by $E_{\rho}$ the minimal conditional expectation, and by $\Phi_{\rho}=\rho^{-1} \circ E_{\rho}$ the minimal left inverse corresponding to $E_{\rho}$. In particular the minimal left inverse of $\varphi$ introduced above is given by

$$
\Phi_{\varphi}: X \rightarrow \frac{1}{d} \sum_{i=1}^{d} T_{i}^{*} X T_{i}, X \in M .
$$

As usual, we define the minimal index $\operatorname{Ind}(\rho)$ as the index of $E_{\rho}$, and the dimension of $\rho$ by $d(\rho)=\sqrt{\operatorname{Ind}(\rho)}$. It turns out that the dimension $d$ is a (well defined) function $\operatorname{Sect}_{0}(M) \rightarrow[1, \infty$ ) which is additive and multiplicative [27].

If $M$ is a factor and $\rho$ is irreducible $\left(\rho(M)^{\prime} \cap M=\mathrm{C}\right)$ with finite index, $\bar{\rho}$ is characterized (modulo inners) by the existence of an isometry $w \in(\mathrm{id}, \rho \bar{\rho})$ (unique up to a phase). If $\rho$ is just of finite index, then $\bar{\rho}$ is characterized by the existence of two isometries $\bar{w} \in(\mathrm{id}, \bar{\rho} \rho), w \in(\mathrm{id}, \rho \bar{\rho})$ such that

$$
\rho\left(\bar{w}^{*}\right) w=d(\rho)^{-1} I=\bar{\rho}\left(w^{*}\right) \bar{w} .
$$

Then

$$
\Phi_{\rho}()=\bar{w}^{*} \bar{\rho}() \bar{w} .
$$

Given a factor $M$ and an endomorphism $\rho \in \operatorname{End}(M)$, we say that $\rho$ is braided if there exists a unitary $\epsilon \in\left(\rho^{2}, \rho^{2}\right)$ such that $\epsilon \rho(\epsilon) \epsilon=\rho(\epsilon) \epsilon \rho(\epsilon)$ [27], Section 3. Furthermore we say that the pair $(\rho, \epsilon)$ is standard if $\rho$ has finite index and $\left\|\Phi_{\rho}(\epsilon)\right\|=d(\rho)^{-1}$. In the sequel we say that $\rho$ is $\epsilon$-braided if the pair $(\rho, \epsilon)$ is standard braided in the above sense.

To conclude, we remark that we only deal with the Hiai-Kosaki-Longo minimal index of the inclusion $\rho(M) \subset M$ associated to the normal extension $\rho$ of an endomorphism $\lambda_{U}$ of the Cuntz algebra to the type III $_{1 / d}$ factor $M$. Equally well, one could also consider the Watatani index relative to some inclusion $\lambda_{U}\left(\mathcal{O}_{d}\right) \subset \mathcal{O}_{d}$ of finite index type [39]. If the minimal conditional expectation $E_{0}: \mathcal{O}_{d} \rightarrow \lambda_{U}\left(\mathcal{O}_{d}\right)$ ([39], Theorem 2.12.3) extends to a normal faithful conditional expectation $E: M \rightarrow \rho(M)$ we have an obvious inequality

$$
\operatorname{Ind}(\rho) \leq \operatorname{Index} E_{0}
$$

[39], Section 2.5. However, there are endomorphisms of the Cuntz algebra which extend to automorphisms of the factor $M$ [1]. 


\section{Yang-Baxter equation and braided endomorphisms}

In this section we study conditions ensuring that an irreducible endomorphism $\lambda_{Y}$ of the Cuntz algebra, with $Y \in\left(H^{2}, H^{2}\right)$ a YB unitary, is $Y$-braided. We prove that if a suitable power $\lambda_{Y}^{n+1}$ of $\lambda_{Y}$ contains an automorphism $\alpha \in \operatorname{Aut}(M)$ of the type-III factor $M$ commuting with $\lambda_{Y}$, then $\lambda_{Y}$ is $Y$ braided. This case covers examples of endomorphisms which are expected to be braided such as the case where $\lambda_{Y}$ is essentially self-conjugate (i.e. $\left(\alpha, \lambda_{Y}^{2}\right) \neq 0$ for some $\left.\alpha \in \operatorname{Aut}(M)\right)$ and the case where a suitable power $n$ of $\lambda_{Y}$ contains its conjugate endomorphism $\left(\left(\mathrm{id}, \lambda_{Y}^{n+1}\right) \neq 0\right)$. We prefer to prove this result first for the above two pivotal particular cases to make things easier.

We start with some elementary lemmas, then we formulate our main results.

Lemma 1. Let $Y \in \mathrm{YB}\left(\mathcal{O}_{d}\right), \alpha \in \operatorname{Aut}(M)$. Then $w \in\left(\alpha^{-1}, \lambda_{Y}^{2}\right)$ if and only if $w$ satisifies

$$
\varphi(Y) Y \varphi(w)=w U
$$

where $U:=\sum_{i=1}^{d} \alpha^{-1}\left(T_{i}\right) T_{i}^{*}$.

Proof. The assertion follows from $\alpha^{-1}(T)=U T, \quad T \in H$.

If $w \in\left(\alpha^{-1}, \lambda_{Y}^{2}\right)$, then $w \alpha^{-1}(T)=\lambda_{Y}(Y) Y T w \equiv \varphi(Y) Y T w$ on $H$, due to Yang-Baxter property; multiplying on the left by $T^{*}$ and summing over an orthonormal basis of $H$ one obtains (1). Conversely, (1) implies that $w \alpha^{-1}(A)=\lambda_{Y}^{2}(A) w$ on a weakly dense subset of $M$.

Lemma 2. Let $\alpha, Y$ be as above. If $\lambda_{Y}$ is irreducible then

$$
\begin{aligned}
Y w & =z_{1} w \\
Y^{*} w & =z_{2} w
\end{aligned}
$$

for some $z_{1}, z_{2} \in \mathrm{T}$.

Proof. In this case $\left(\alpha^{-1}, \lambda_{Y}^{2}\right)$ is one dimensional [8], and by the YB equation both $w_{1}:=Y w, w_{2}:=Y^{*} w$ satisfy (1).

Next result shows that YB endomorphisms are braided under natural additional assumptions.

TheOREM 1. Let $Y \in \mathrm{YB}\left(\mathcal{O}_{d}\right)$ with $\rho:=\lambda_{Y} \in \operatorname{End}(M)$ irreducible and essentially self-conjugate.

Then $\rho$ is $Y$-braided.

Proof. We have to prove that $d(\rho)=\left|\Phi_{\rho}(Y)\right|^{-1}$ where $\Phi_{\rho}$ is the unique left inverse of $\rho$. 
From $\rho^{2} \alpha \succ \mathrm{id}, \alpha \rho^{2} \succ \mathrm{id}$, it follows that $\rho \alpha, \alpha \rho$, are both conjugate for $\rho$, namely $\rho \alpha=\operatorname{ad} V \alpha \rho$ for some unitary $V \in M$; one has

$$
\begin{aligned}
\rho \alpha \rho(T) V \alpha(w) & =V \alpha \rho^{2}(T) \alpha(w) \\
& =V \alpha\left(\rho^{2}(T) w\right) \\
& =V \alpha\left(w \alpha^{-1}(T)\right) \\
& =V \alpha(w) T .
\end{aligned}
$$

Hence the conjugate isometry is given by $\bar{w}=V \alpha(w)$ [26]. After some simplifications one obtains, for the left inverse of $\rho$,

$$
\Phi_{\rho}(\cdot)=\alpha\left(w^{*} \rho(\cdot) w\right) .
$$

Since $w w^{*}$ is the Jones' projection for the inclusion $\rho \bar{\rho}(M) \subset \rho(M)$, the statistical dimension is given by $d(\rho)^{-2}=E_{\rho}\left(w w^{*}\right)$ [26]. Moreover $w w^{*} \in$ $(\rho \bar{\rho}, \rho \bar{\rho}) \equiv\left(\rho^{2}, \rho^{2}\right) \subset\left(H^{2}, H^{2}\right)$, see [3], Proposition 4.2, hence $\rho\left(w w^{*}\right)=$ $Y \varphi(Y) w w^{*} \varphi\left(Y^{*}\right) Y^{*}$, see [5], Proposition 1.2. One obtains

$$
\begin{aligned}
E_{\rho}\left(w w^{*}\right) & \equiv \rho \Phi_{\rho}\left(w w^{*}\right) \\
& =\rho \alpha\left(w^{*} \rho\left(w w^{*}\right) w\right) \\
& =\operatorname{ad} V \alpha \rho\left(w^{*} \rho\left(w w^{*}\right) w\right) \\
& \equiv \rho\left(w^{*} \rho\left(w w^{*}\right) w\right) \\
& =: B B^{*}
\end{aligned}
$$

where $B=w^{*} Y \varphi(Y) w$. Due to the Yang-Baxter equation together with Lemma 2, we have $B=\varphi\left(w^{*} Y\right) Y^{*} w=\varphi\left(w^{*}\right) w$. We note that $\Phi_{\rho}(Y) \in(\rho, \rho) \equiv \mathrm{C} I$ by irreducibility, thus from (1), Lemma 2 and YangBaxter property it follows that $\varphi\left(w^{*}\right) w=\alpha \Phi_{\rho}(Y)$. Finally we obtain $d(\rho)^{-2}=B B^{*}=\left|\Phi_{\rho}(Y)\right|^{2}$.

COROLlaRY 1. Under the same hypotheses of the preceding theorem, we have

$$
d(\rho)=|\tau(Y)|^{-1}
$$

where $\tau$ is the normalized trace on $\left(H^{2}, H^{2}\right)$.

Proof. $Y \in\left(H^{2}, H^{2}\right) \Rightarrow \lambda_{Y} \circ \sigma_{t}=\sigma_{t} \circ \lambda_{Y}$ and the unique left inverse $\Phi_{\rho}$ of $\lambda_{Y}$ satisfies $\omega \circ \Phi_{\rho}=\omega$ ([3], Lemma 3.2). Hence $\Phi_{\rho}(Y)=\omega\left(\Phi_{\rho}(Y) I\right) \equiv$ $\omega\left(\Phi_{\rho}(Y)\right)=\omega(Y)$ and $\omega$ reduces to the normalized trace on $\left(H^{2}, H^{2}\right)$.

For completeness we recall the following well-known elementary results. 
Lemma 3. Let $Y \in \mathrm{YB}\left(\mathcal{O}_{d}\right)$. Then

(i) $\lambda_{Y}^{n}(Y)=\varphi^{n}(Y)$.

(ii) $\lambda_{Y}^{n}=\lambda_{U_{n}}$ where $U_{n}=\varphi^{n-1}(Y) \cdots \varphi(Y) Y$.

Proof. It follows by induction applying repeatedly the YB property.

Let $Y \in \mathrm{YB}\left(\mathcal{O}_{d}\right)$ with $\rho:=\lambda_{Y}$ an irreducible endomorphism of $M$ and suppose that $\rho^{n+1}$ contains the identity sector i.e. $\left(\mathrm{id}, \rho^{n+1}\right) \neq \emptyset$.

LEMMA 4. The following two statements hold.

(i) $v \in\left(\mathrm{id}, \rho^{n+1}\right)$ if and only if $v$ satisfies

$$
\varphi^{n}(Y) \cdots \varphi(Y) Y \varphi(v)=v .
$$

(ii) The free algebra generated by $\left\{\varphi^{k}(Y), \varphi^{l}\left(Y^{*}\right) ; k, l=0,1, \ldots, n-1\right\}$ acts on the finite dimensional Hilbert space (id, $\left.\rho^{n+1}\right)$.

Proof. (i) It follows easily from Lemma 1.

(ii) If $k<n$ we have

$$
\begin{aligned}
& \varphi^{n}(Y) \cdots \varphi(Y) Y \varphi\left(\varphi^{k}\left(Y^{\sharp}\right) v\right)= \\
& \varphi^{n}(Y) \cdots \varphi^{k+1}(Y) \varphi^{k}(Y) \varphi^{k+1}\left(Y^{\sharp}\right) \varphi^{k-1}(Y) \cdots \varphi(Y) Y \varphi(v)= \\
& \varphi^{n}(Y) \cdots \varphi^{k}\left(Y^{\sharp}\right) \varphi^{k+1}(Y) \varphi^{k}(Y) \varphi^{k-1}(Y) \cdots \varphi(Y) Y \varphi(v)= \\
& \varphi^{k}\left(Y^{\sharp}\right) \varphi^{n}(Y) \cdots \varphi(Y) Y \varphi(v)=\varphi^{k}\left(Y^{\sharp}\right) v
\end{aligned}
$$

and the assertion follows by (i).

The following result can be considered as a generalization of Theorem 1 .

TheOREM 2. Let $Y \in \mathrm{YB}\left(\mathcal{O}_{d}\right)$ with $\rho:=\lambda_{Y}$ an irreducible sector such that $\rho^{n+1}$ contains the identity sector.

Then $\lambda_{Y}$ is $Y$-braided.

Proof. Let $v \in\left(\mathrm{id}, \rho^{n+1}\right)$ and $p:=s_{\left(\rho^{n}, \rho^{n}\right)}\left(v v^{*}\right)$ the support of $v v^{*}$ in the algebra $\left(\rho^{n}, \rho^{n}\right)$, then we get for $\bar{\rho}$

$$
\bar{\rho}(\cdot)=u^{*} \rho^{n}(\cdot) u
$$

where $u$ is an isometry with $u u^{*}=p$ [10]. Besides we get $w \in(\mathrm{id}, \rho \bar{\rho})$, $\bar{w} \in(\mathrm{id}, \bar{\rho} \rho)$ where

$$
w=\rho\left(u^{*}\right) v, \quad \bar{w}=u^{*} v .
$$

We have $\Phi_{\rho}(Y)=v^{*} u u^{*} \rho^{n}(Y) u u^{*} v=v^{*} \varphi^{n}(Y) v$. However 


$$
\begin{aligned}
d(\rho)^{-2} & =E_{\bar{\rho}}\left(\bar{w} \bar{w}^{*}\right) \\
& =\bar{\rho}\left(v^{*} \rho(u) \rho\left(u^{*} v v^{*} u\right) \rho\left(u^{*}\right) v\right) \\
& =\bar{\rho}\left(v^{*} \rho\left(v v^{*}\right) v\right)
\end{aligned}
$$

Since $v v^{*}$ is an element of degree 0 in $\left(\rho^{n+1}, \rho^{n+1}\right)$, then $v v^{*} \in\left(H^{n+1}, H^{n+1}\right)$ ([3] Proposition 4.2) and

$$
\rho\left(v v^{*}\right)=\operatorname{ad} Y \operatorname{ad} \varphi(Y) \cdots \operatorname{ad} \varphi^{n}(Y)\left(v v^{*}\right)
$$

([5], Proposition 1.2). Therefore we have $E_{\bar{\rho}}\left(\bar{w} \bar{w}^{*}\right)=\bar{\rho}\left(B B^{*}\right)$ where $B:=$ $v^{*} Y \cdots \varphi^{n}(Y) v$.

As we have explained in Lemma 4, part (ii), the operator

$$
V_{n}:=\varphi^{n-1}\left(Y^{*}\right) \cdots \varphi\left(Y^{*}\right) Y^{*}
$$

is a unitary acting on (id, $\rho^{n+1}$ ) hence it is diagonalizable. Then we can choose $v \in\left(\mathrm{id}, \rho^{n+1}\right)$ which is an eigenvector for $V_{n}$.

The conclusion now follows by

$$
\begin{aligned}
d(\rho)^{-2} & =E_{\bar{\rho}}\left(\bar{w} \bar{w}^{*}\right) \\
& =\bar{\rho}\left(\left|\Phi_{\rho}(Y)\right|^{2} I\right) \\
& =\left|\Phi_{\rho}(Y)\right|^{2} I .
\end{aligned}
$$

The proof of Theorem 1 and Theorem 2 above can be generalized in order to include the following case.

THEOREM 3. Let $Y \in \mathrm{YB}\left(\mathcal{O}_{d}\right)$ with $\rho:=\lambda_{Y}$ an irreducible endomorphism such that $\rho^{n+1}$ contains an automorphism $\alpha^{-1}$ of $M$ commuting with $\rho$ in $\operatorname{Sect}(M)$.

Then $\lambda_{Y}$ is $Y$-braided.

Proof. First note that, as in the previous cases, the free algebra generated by $\left\{\varphi^{k}(Y), \varphi^{l}\left(Y^{*}\right) ; k, l=0,1, \ldots, n-1\right\}$ acts on the finite dimensional Hilbert space $\left(\alpha^{-1}, \rho^{n+1}\right)$. Let $v \in\left(\alpha^{-1}, \rho^{n+1}\right)$ be an isometry. Now we compute a conjugate for $\rho$ as a subsector of $\rho^{n} \alpha$ and the corresponding isometries $w, \bar{w}$ [10]. By hypothesis we have $\rho \alpha=\operatorname{ad}(V) \alpha \rho$ for some unitary $V$ in $M$, hence $\rho^{n}\left(V^{*}\right) v \in\left(\mathrm{id}, \rho^{n} \alpha \rho\right)$. Putting $p:=s_{\left(\rho^{n}, \rho^{n}\right)}\left(v v^{*}\right)$ we get

$$
\bar{\rho}(\cdot)=u^{*} \rho^{n} \alpha(\cdot) u
$$

where $u$ is an isometry with $u u^{*}=p$. We have for $w, \bar{w}$ the following formulas:

$$
w=\rho\left(u^{*}\right) v, \quad \bar{w}=u^{*} \rho^{n}\left(V^{*}\right) v .
$$

After straightforward calculations we obtain $\Phi_{\rho}(Y)=\alpha\left(v^{*} \varphi^{n}(Y) v\right)$ and 


$$
\begin{aligned}
d(\rho)^{-2} & \equiv E_{\bar{\rho}}\left(\bar{w} \bar{w}^{*}\right) \\
& =\bar{\rho} \operatorname{ad}\left(\alpha^{-1}\left(V^{*}\right)\right)\left(v^{*} \rho\left(v v^{*}\right) v\right) .
\end{aligned}
$$

The proof now follows as in the last theorem.

REMARK 1. Let $\lambda_{Y}$ be an irreducible YB endomorphism. If we set

$$
\pi\left(\sigma_{i}\right):=\lambda_{Y}^{i-1}(Y), \quad i=1,2, \ldots,
$$

where $\sigma_{i}$ are the generators of the Artin braid group $\mathbf{B}_{\infty}$, then we have a unitary representation $\pi$ of $\mathrm{B}_{\infty}$ and a link invariant determined by a usual Markov trace procedure (see [27], Proposition 3.1) which is naturally attached to the endomorphism $\lambda_{Y}$.

As we have explained in the Introduction, in the standard case (i.e. when $\lambda_{Y}$ is $Y$-braided), these invariants seem to have an important role for the theory of dimension in $C^{*}$-category $([27,29])$, as well as for relevant applications in low-dimensional QFT ([11, 26, 31, 34, 36]).

For low-channel sectors, it is well known that these representations factorize via Hecke algebras or Birman-Wenzl-Murakami algebras representations uniquely determinated by the dimension $d\left(\lambda_{Y}\right)$ up to tensorization with a one-dimensional representation of $\mathrm{B}_{\infty}$, see [27], Theorem 3.2, see also [11, 35]. In the standard case we also have

$$
\left[\mathscr{M}: \lambda_{Y}(\mathscr{M})\right]=d\left(\lambda_{Y}\right)^{2},
$$

where $\mathscr{M}:=\pi_{\tau}\left(\cup_{n}\left(\lambda_{Y}^{n}, \lambda_{Y}^{n}\right)\right)^{\prime \prime}$ is the hyperfinite type $\mathrm{II}_{1}$-algebra generated by the tower of relative commutants (where $\tau$ is the canonical faithful Markov trace on $\cup_{n}\left(\lambda_{Y}^{n}, \lambda_{Y}^{n}\right)$ obtained via the left inverse), see [11].

To conclude this section, we give a sufficient condition for Yang-Baxter endomorphisms of index 4 of $\mathcal{O}_{2}$ to be braided. Then we exhaust all the cases appearing in $\mathrm{O}_{2}$, see [3], Corollary 7.6. As in [3], Section 7 we define

$$
S:=\frac{1}{\sqrt{2}}\left(T_{1} T_{2}-T_{2} T_{1}\right)
$$

Proposition 1. Let $Y \in \mathrm{YB}\left(\mathrm{O}_{2}\right)$. Suppose that there exists a unitary $\bar{Y} \in\left(H^{2}, H^{2}\right)$ (not necessarily $Y B$ ) such that

(i) $\varphi(Y) \bar{Y} \varphi(S)=\varphi(\bar{Y}) Y \varphi(S)=S$,

(ii) $\bar{Y} S=\beta S$ for some $\beta \in \mathrm{T}$.

Then $\lambda_{Y}$ is $Y$-braided.

Proof. Denoting by $\Phi_{\rho}$ (resp. $E_{\rho}$ ) the minimal left inverse (resp. the minimal conditional expectation) of $\rho:=\lambda_{Y}$, we have to prove that 


$$
\left\|\Phi_{\rho}(Y)\right\|^{-1}=d(\rho)
$$

where the r.h.s. is 2 by [3], Corollary 7.6.

From $\bar{\rho}=\lambda_{\bar{Y}}, \bar{w}=-\bar{Y} S$ (see [3], Corollary 7.6), and condition (i), we get

$$
d(\rho)^{-2}=E_{\rho}\left(Y S S^{*} Y^{*}\right)=\rho\left(\varphi\left(S^{*}\right) S S^{*} \varphi(S)\right) .
$$

On the other hand

$$
\Phi_{\rho}(Y)=\varphi\left(S^{*}\right) \varphi\left(\bar{Y}^{*}\right) S .
$$

The proof now follows from the condition (ii).

The above results suggest that non-trivial endomorphisms $\lambda_{Y}$ of Cuntz algebras which are standard braided with respect to $Y$ could exist. In the following section we show that this is in fact the case, studying some properties of the endomorphisms of $\mathcal{O}_{2}$ associated with Yang-Baxter unitaries.

\section{Examples of braided endomorpisms of $\mathcal{O}_{2}$}

In this section we discuss explicit examples of braided endomorphisms of the unique AFD factor of type $\mathrm{III}_{\frac{1}{2}}$ associated to solutions of the $\mathrm{YB}$ equation on a Hilbert space of dimension 2 , see [15].

In the following we denote by $\varepsilon \in \mathscr{M}_{2}$ the $4 \times 4$ YB unitary given by

$$
\varepsilon:=\frac{1}{\sqrt{2}}\left(\begin{array}{cccc}
1 & \cdot & \cdot & 1 \\
\cdot & 1 & 1 & \cdot \\
\cdot & -1 & 1 & \cdot \\
-1 & \cdot & \cdot & 1
\end{array}\right) .
$$

Then $\varepsilon=R F$, with $R$ as in the case $R_{H 0.2}$ from [15]. It is useful to write $\varepsilon=\frac{1}{\sqrt{2}}(I+y)$, with $y \in \mathscr{M}_{2}$ the anti-diagonal unitary

$$
y:=\left(\begin{array}{cccc}
\cdot & \cdot & \cdot & 1 \\
\cdot & \cdot & 1 & \cdot \\
\cdot & -1 & \cdot & \cdot \\
-1 & \cdot & \cdot & \cdot
\end{array}\right) .
$$

Clearly $y^{2}=-I$ i.e. $y^{*}=-y$, thus $\varepsilon^{2}=y=\sqrt{2} \varepsilon-I, \varepsilon^{*}=\frac{1}{\sqrt{2}}(I-y)$. It is easy to check that $y \varphi(y) y \varphi\left(y^{*}\right)=I$ i.e. $y \varphi(y)=-\varphi(y) y$. It follows that $\lambda_{y}(y)=y^{*}, \lambda_{y}^{2}=\lambda_{\lambda_{y}(y) y}=$ id thus $\lambda_{y}$ is a localized automorphism of $\mathcal{O}_{2}$ with $\lambda_{y}{ }^{-1}=\lambda_{y}$.

We will see in the next section that (the normal extension of) this automorphism is outer on $M=\pi_{\omega}\left(\mathcal{O}_{2}\right)^{\prime \prime}$. More precisely, our example comes from an outer action $\alpha: Z_{2} \rightarrow \operatorname{Aut}(M)$ with $\lambda_{\varepsilon}(M) \subseteq M^{\alpha} \subset M$ (see below), and therefore $\lambda_{\varepsilon}(M)=M^{\alpha}$ by an obviuos index argument. 
Let us denote by $e_{+}, e_{-}$the two rank-two orthogonal spectral projections of $\varepsilon$ associated to the two eigenvalues $\frac{1}{\sqrt{2}}(1 \pm i)$; then we have

$$
\varepsilon=\frac{1+i}{\sqrt{2}} e_{+}+\frac{1-i}{\sqrt{2}} e_{-},
$$

where $e_{ \pm}$are expressed in terms of $y$ as

$$
e_{+}=\frac{1}{2}(I-i y), \quad e_{-}=\frac{1}{2}(I+i y) .
$$

Moreover both $e_{+}, e_{-}$satisfy the Temperley-Lieb Goldman-type relation [21]

$$
\left(e_{ \pm}-\varphi\left(e_{ \pm}\right)\right)^{2}=\frac{1}{2} I .
$$

The following proposition is a direct consequence of Theorem 1 taking into account the results contained in [3].

Proposition 2. $\operatorname{Ind}\left(\lambda_{\varepsilon}\right)=2=\left|\phi_{\varepsilon}(\varepsilon)\right|^{-2}$. In particular $\lambda_{\varepsilon} \in \operatorname{End}(M)$ is an irreducible localized endomorphism of $M$ which is also essentially self-conjugate and $\varepsilon$-braided.

Proof. From [3], Proposition 8.3 and the discussion above we deduce that $\operatorname{Ind}\left(\lambda_{\varepsilon}\right)=2$ and the proof now follows from Theorem 1 .

Clearly the same arguments may also be applied to $\varepsilon^{*}$, see below. It is worth mentioning that the last result also gives a positive answer to a question raised in [3], see the remark after Proposition 9.9.

REMARK 2. We also stress that, putting $g_{1}:=\frac{i-1}{\sqrt{2}} \varepsilon$, we obtain representations of the Hecke algebras $\mathrm{H}_{n}(q)$ with generators $g_{1}, \ldots, g_{n-1}$ and relations

$$
\begin{gathered}
g_{i} g_{i+1} g_{i}=g_{i+1} g_{i} g_{i+1}, \quad i=1, \ldots, n-2, \\
g_{i} g_{j}=g_{j} g_{i}, \quad|i-j| \geq 2, \\
g_{i}{ }^{2}=(q-1) g_{i}+q,
\end{gathered}
$$

for $q=i$ inside $\mathscr{M}_{n} \simeq \mathrm{M}_{2}(\mathrm{C}) \otimes \ldots \otimes \mathrm{M}_{2}(\mathrm{C})$ ( $n$ times) such that $g_{k+1}=\varphi\left(g_{k}\right)$, $k=1, \ldots, n-2$, see [3], Section 9, for further details. This construction parallels the one given by Pimsner and Popa in the case $q \geq 1$ (see e.g. [21], p.247). We were not able to find it in the existing literature.

Remark 3. One can consider also the self-adjoint projection 


$$
P=\frac{1}{2} \sum_{i, j=1}^{2} e_{i j} \otimes e_{i j}
$$

where $e_{i j}$ are the matrix units in $\mathrm{M}_{2}(\mathrm{C})$.

However, easy calculations show that $P$ together with its shifted projections satisfy the Temperley-Lieb relations with parameter $\frac{1}{4}$, and thus lead to a representation of the Hecke algebra with parameter $q=1$. Namely, such a representation degenerate into a representation of the group algebra of the permutations.

The corresponding solution $R=2 P-1$ of the YB equation falls within the class (4) discussed below, with $a=-1, b=1$. Therefore, the associated endomorphism $\lambda_{R}$ has index 4 . It is also easy to see that $\lambda_{R}$ is reducible and essentially self-conjugate whereas $\lambda_{i R}$ is self-conjugate (details are left to the reader).

REMARK 4. It is well known that, in the situation described in Remark 2,

$$
M \supset \lambda_{\varepsilon}(M) \supset \lambda_{\varepsilon}^{2}(M) \supset \ldots
$$

provides a Jones tunnel with principal graph $A_{3}$. Therefore it is straightforward to see that the algebra generated by $I$ and the Jones projections $e_{+}, \varphi\left(e_{+}\right), \varphi^{2}\left(e_{+}\right), \ldots, \varphi^{n-2}\left(e_{+}\right)$coincides with $\left(\lambda_{\varepsilon}^{n}, \lambda_{\varepsilon}^{n}\right)$ just by computing the corresponding dimensions. In particular $\left(\lambda_{\varepsilon}^{n}, \lambda_{\varepsilon}^{n}\right) \subset\left(H^{n}, H^{n}\right)$, thus for any $X \in\left(\lambda_{\varepsilon}^{n}, \lambda_{\varepsilon}^{n}\right), n \geq 1$ we have

$$
\lambda_{\varepsilon}(X)=\varepsilon \varphi(\varepsilon) \ldots \varphi^{n-1}(\varepsilon) X \varphi^{n-1}(\varepsilon)^{*} \ldots \varphi(\varepsilon)^{*} \varepsilon^{*} .
$$

We now discuss other related examples. We start recalling the following well-known

Lemma 5. Let $Y \in\left(H^{2}, H^{2}\right) \subset \mathcal{O}_{d}$ a YB unitary.

Then $Y_{Q}:=(Q \otimes Q) Y(Q \otimes Q)^{*}, Y^{*}, Y^{t}, \Delta Y \Delta, F Y F$ are all YB unitaries, where $Q \in U(H)$, and $\Delta$ is the matrix with 1 on the anti-diagonal and 0 elsewhere.

Thus, starting from $\varepsilon$, we can also consider
(a) $\varepsilon_{Q}=\frac{1}{\sqrt{2}}\left(I+(Q \otimes Q) y(Q \otimes Q)^{*}\right)$,
(b) $\varepsilon^{*}=\Delta \varepsilon \Delta$,
(c) $F \varepsilon F=\left(\begin{array}{cccc}1 & \cdot & \cdot & 1 \\ \cdot & 1 & -1 & \cdot \\ \cdot & 1 & 1 & \cdot \\ -1 & \cdot & \cdot & 1\end{array}\right)$

which are precisely obtained applying to $\varepsilon$ the transformations considered in [15], pag. 246. 
Many properties of the corresponding endomorphisms can be deduced by the following

Proposition 3. Let $Y$ be a unitary element in $\left(H^{2}, H^{2}\right)$. Then

$$
\operatorname{Ind}\left(\lambda_{Y_{Q}}\right)=\operatorname{Ind}\left(\lambda_{Y}\right) \text {. }
$$

Moreover:

If $\lambda_{Y}$ is irreducible, then $\lambda_{Y_{Q}}$ is irreducible as well. Furthermore $\lambda_{Y_{Q}}$ is selfconjugate if and only if $\lambda_{Y}$ is.

If $Y$ is a $\mathrm{YB}$ unitary and $d=2$, then

$$
\operatorname{Ind}\left(\lambda_{Y^{*}}\right)=\operatorname{Ind}\left(\lambda_{F Y F}\right)=\operatorname{Ind}\left(\lambda_{Y}\right) .
$$

If in addition $\operatorname{Ind}\left(\lambda_{Y}\right)=2$, then $\lambda_{Y}, \lambda_{Y_{Q}}, \lambda_{Y^{*}}$ and $\lambda_{F Y F}$ are all braided.

Proof. Note that, with the notation as above, we have

$$
\lambda_{Y_{Q}}=\lambda_{Q \varphi(Q) Y Q^{*} \varphi\left(Q^{*}\right)}=\lambda_{Q} \lambda_{Y} \lambda_{Q^{*}}
$$

Therefore the first identity is obviuos since the index is multiplicative and $\lambda$ restricts on $U(H)$ to a group homomorphism into $\operatorname{Aut}(M)$. The second statement follows from $F Q \otimes Q=Q \otimes Q F$, see [3], Corollary 4.3. Passing to equivalence classes it is easy to see that also the third statement is true. The next statement can be checked looking at the three possible cases. In fact if $d=2$, all the indices are in the set $\{1,2,4\}$ [3], Proposition 9.9. If $\operatorname{Ind}\left(\lambda_{Y}\right)=1$ i.e. $\lambda_{Y}$ is an automorphism using the fact that the YB equation is equivalent to $Y \in\left(\lambda_{Y}^{2}, \lambda_{Y}^{2}\right)$ we deduce that $Y \in \mathrm{C} I$, therefore $Y=F Y F$ and $Y^{*} \in \mathrm{C} I$ too, hence $\operatorname{Ind}\left(\lambda_{Y^{*}}\right)=\operatorname{Ind}\left(\lambda_{F Y F}\right)=1$. If $\operatorname{Ind}\left(\lambda_{Y}\right)=2$ then the two rank-two spectral projections $e_{+}=e, e_{-}=I-e$ of $Y$ both satisfy the Temperley-Lieb relations as above thus the same relations hold for the two spectral projections of $Y^{*}$ and $F Y F$, therefore $\operatorname{Ind}\left(\lambda_{Y^{*}}\right)=\operatorname{Ind}\left(\lambda_{F Y F}\right)=2$. Finally in this case $\lambda_{Y}, \lambda_{Y_{Q}}, \lambda_{Y^{*}}$ and $\lambda_{F Y F}$ are essentially self-conjugate [3], Proposition 8.3 , thus they are also standard braided by Theorem 1 .

Now we consider some YB unitaries giving rise to endomorphisms of $\mathrm{O}_{2}$ of index 4. These unitaries correspond precisely to the classes $R_{H 3.1}, R_{H 1.4}$ classified by [15] respectively; the transformations contained in [15], pag. 246, cause no further complications.

We start with a three-parameter family of matrices

$$
Y=\left(\begin{array}{cccc}
1 & \cdot & \cdot & \cdot \\
\cdot & \cdot & a & \cdot \\
\cdot & b & \cdot & \cdot \\
\cdot & \cdot & \cdot & c
\end{array}\right)
$$


where $a, b, c \in \mathrm{T}$ and we have scaled the first entry by multiplication by a phase factor.

Bearing in mind that the matrix entries $R_{i j}^{k l}$ given in the formula (4) of [15] correspond, in the Cuntz algebras formalism, to the elements $R_{i j}^{k l} T_{j} T_{i} T_{k}^{*} T_{l}^{*}$, one can compute the formula for the unitary $\bar{Y}$ implementing the conjugate endomorphism of $\lambda_{Y}$ taking into account condition (i) of Proposition 1. We obtain

$$
\bar{Y}=\bar{b}\left(\begin{array}{cccc}
1 & \cdot & \cdot & \cdot \\
\cdot & \cdot & \frac{b}{c} & \cdot \\
\cdot & b & \cdot & \cdot \\
\cdot & \cdot & \cdot & \frac{b}{a}
\end{array}\right) .
$$

Applying (ii) of Proposition 1, we can easily compute the conditions under which $\lambda_{Y}$, or both $\lambda_{Y}, \lambda_{\bar{Y}}$ are standard braided.

We summarize these results in the following

Proposition 4. Let $Y$ be a $\mathrm{YB}$ unitary belonging to the family (2). Then we have

(i) $\lambda_{Y}$ is $Y$-braided if and only if $c=1$,

(ii) $\lambda_{Y}$ is $Y$-braided and $\lambda_{\bar{Y}}$ is $\bar{Y}$-braided at the same time if and only if $c=1$ and $a=b$.

The same considerations can be applied to the case corresponding to the two-parameter family of matrices

$$
Y=\left(\begin{array}{cccc}
\cdot & \cdot & \cdot & 1 \\
\cdot & a & \cdot & \cdot \\
\cdot & \cdot & a & \cdot \\
b & \cdot & \cdot & \cdot
\end{array}\right)
$$

obtaining

$$
\bar{Y}=-\bar{b}\left(\begin{array}{cccc}
\cdot & \cdot & \cdot & 1 \\
\cdot & \frac{b}{a} & \cdot & \cdot \\
\cdot & \cdot & \frac{b}{a} & \cdot \\
b & \cdot & \cdot & \cdot
\end{array}\right)
$$

In such a situation we have

Proposition 5. Let $Y$ be a YB unitary belonging to the family (4).

Then $\lambda_{Y}$ is $Y$ braided and $\lambda_{\bar{Y}}$ is $\bar{Y}$-braided as well.

To conclude this section we note that the conjugation map $Y \rightarrow \bar{Y}$ given by formulas (3), (5) respectively, is involutive on the YB unitaries giving rise to endomorphisms of index 4. 


\section{Some fusion rules}

Now we compute the fusion rules in $\operatorname{Sect}(M)=\operatorname{End}(M) / \operatorname{Inn}(M)$ associated to $\rho_{0}=\mathrm{id}, \rho_{\frac{1}{2}}=\lambda_{\varepsilon}$ and $\rho_{1}=\lambda_{y}$.

We begin by noticing that [1] is not inner: in fact it is easy to see that $\lambda_{y} \simeq \lambda_{\tilde{y}}$, where $\tilde{y}=\left(\begin{array}{cc}-1 & 0 \\ 0 & 1\end{array}\right)$, and the last automorphism is outer since, otherwise, it should be given by the adjoint action of a unitary in $M$ commuting with $T_{1}$ and anticommuting with $T_{2}$, which is not possible.

For completeness we report the following result, probably well-known to experts, which can be immediately deduced e.g. from [17], Proposition 4.5.

Proposition 6. Given any $\lambda_{U} \in \operatorname{Aut}(M)$ with $U \in(H, H), U \neq I$, then its equivalence class (modulo inners) is not trivial in $\operatorname{Out}(M)$.

Now we have

$$
[\overline{0}]=[0], \quad[\overline{1}]=\left[\left(\lambda_{y}\right)^{-1}\right]=[1]
$$

and

$$
[0][i]=[i][0], \quad i=0, \frac{1}{2}, 1
$$

$$
[1]^{2}=[0]
$$

Furthermore

$$
[1]\left[\frac{1}{2}\right]=\left[\frac{1}{2}\right]=\left[\frac{1}{2}\right][1],
$$

implying

$$
[1]\left[\frac{\overline{1}}{2}\right]=\left[\frac{1}{2}\right]=\left[\frac{1}{2}\right][1] .
$$

Indeed, the first equality in (iii) is a consequence of $\lambda_{y} \lambda_{\varepsilon}=\lambda_{\lambda_{y}(\varepsilon) y}$ and $\lambda_{y}(\varepsilon) y=\lambda_{y}\left(\frac{I+y}{\sqrt{2}}\right) y=\frac{I+y^{*}}{\sqrt{2}} y=\varepsilon$, and the second one follows from $\lambda_{\varepsilon} \lambda_{y}=$ $\lambda_{\lambda_{\varepsilon}(y) \varepsilon}=\lambda_{\varphi(y) \varepsilon}=\operatorname{ad}\left(y^{*}\right) \lambda_{\varepsilon} \simeq \lambda_{\varepsilon}$, where we used that $\varphi(y) \varepsilon=y^{*} \varepsilon \varphi(y)$. These relations finally imply

$$
\left[\frac{1}{2}\right]\left[\frac{1}{2}\right]=\left[\frac{1}{2}\right]\left[\frac{1}{2}\right]=[0] \oplus[1] .
$$

It is natural to ask if $\left[\frac{1}{2}\right]=\left[\frac{1}{2}\right]$ because of some similarity with the representation category of $\mathscr{U}_{-i}(\operatorname{sl}(2))$, see e.g. [30]. However we prove that in fact this is not true.

Proposition 7. $\lambda_{\varepsilon}^{2}$ does not contain any modular automorphism. 
Proof. We show that it is not possible to find an isometry $v \in\left(\lambda_{z I}, \lambda_{\varepsilon}^{2}\right)$, $z \in \mathrm{T}$. Let us assume the converse, then we have

$$
\begin{aligned}
v z T_{i} & =\varphi(\varepsilon) \varepsilon T_{i} v \\
& =\left(I-\varepsilon^{*} \varphi(\varepsilon)^{*}\right) T_{i} v \\
& =T_{i} v-\varepsilon^{*} T_{i} \varepsilon^{*} v \\
& =\left(I-\frac{1 \mp i}{\sqrt{2}} \varepsilon^{*}\right) T_{i} v \\
& =\left(I-\frac{1 \mp i}{2}(I-y)\right) T_{i} v \\
& =\left(\frac{1 \pm i}{2} I+\frac{1 \mp i}{2} y\right) T_{i} v \\
& =\frac{1 \pm i}{2}(I \mp i y) T_{i} v \\
& =(1 \pm i) e_{ \pm} T_{i} v,
\end{aligned}
$$

and finally $z v=(1 \pm i) e_{ \pm} \varphi(v)$, where we used that $\varepsilon^{*} v=\varepsilon^{*} v v^{*} v=\frac{1 \mp i}{\sqrt{2}} v$ and the sign is chosen according to $v v^{*}=e_{+}$or $v v^{*}=e_{-}$respectively.

It follows that $z v \varphi\left(v^{*}\right)=(1 \pm i) e_{ \pm} \varphi\left(e_{ \pm}\right)$, thus

$$
\begin{aligned}
z \Phi_{\varphi}(v) v^{*} & =(1 \pm i) \Phi_{\varphi}\left(e_{ \pm}\right) e_{ \pm} \\
& =\frac{1 \pm i}{2} e_{ \pm} \\
& =\frac{1 \pm i}{2} v v^{*}
\end{aligned}
$$

since $\Phi_{\varphi}(y)=0$. Hence the relation $z \Phi_{\varphi}(v)=\frac{1 \pm i}{2} v$ should hold.

Note that, according to [3], Proposition 4.2, such an isometry $v$ should be contained in $\left(H^{2}, H^{2}\right) \oplus\left(H, H^{2}\right) \oplus H^{2}$. Applying sufficiently many times $\Phi_{\varphi}$ to $v$ we are led to consider $v \in \mathrm{C} \oplus H \oplus H^{2}$.

The conclusion is easily deduced observing that the minimal left inverse $\Phi_{\varphi}$ of $\varphi$ acts as $\frac{1}{2} I$ on $H$, and as $\frac{1}{2} F$ on $H^{2}$.

Now we investigate about the structure of the ${ }^{*}$-semiring generated by $\rho \equiv \lambda_{\varepsilon}$ in $\operatorname{Sect}(M)$. Recall among others the relation $\left[\frac{1}{2}\right]^{2}=\alpha_{1}+\alpha_{2}$ (with $\left.\alpha_{1} \neq \alpha_{2}\right)$.

Lemma 6. We have

$$
\begin{aligned}
\alpha_{1} \alpha_{2}^{-1} & =\alpha_{2} \alpha_{1}^{-1}=[1], \\
\alpha_{1} \alpha_{2} & =\alpha_{2} \alpha_{1}, \\
\alpha_{1}^{2} & =\alpha_{2}^{2} .
\end{aligned}
$$


Moreover $\alpha_{i}, i=1,2$ commute with $\left[\frac{1}{2}\right],\left[\frac{1}{2}\right]$ and $[1]$.

ProOF. $\left[\frac{1}{2}\right]=\alpha_{1}^{-1}\left[\frac{1}{2}\right]=\left[\frac{1}{2}\right] \alpha_{1}^{-1}=\alpha_{2}^{-1}\left[\frac{1}{2}\right]=\left[\frac{1}{2}\right] \alpha_{2}^{-1}, \quad$ thus $\quad[0] \oplus[1]=\left[\frac{1}{2}\right]\left[\frac{1}{2}\right]=$ $[0] \oplus \alpha_{1} \alpha_{2}^{-1}=[0] \oplus \alpha_{2} \alpha_{1}^{-1}=\left[\frac{1}{2}\right]\left[\frac{1}{2}\right]=[0] \oplus \alpha_{1}^{-1} \alpha_{2}=[0] \oplus \alpha_{2}^{-1} \alpha_{1}$.

Therefore $\alpha:=\alpha_{1}$ and $\beta:=[1]$ generate, inside $\operatorname{Sect}(M)$, a subgroup $\langle\alpha, \beta\rangle$ which is isomorphic to $\mathbf{Z}_{\operatorname{ord}\left(\alpha_{1}\right)} \times \mathbf{Z}_{2}$. In fact it is not difficult to show that $\operatorname{ord}\left(\alpha_{1}\right)=\infty$ thus $\langle\alpha, \beta\rangle=\mathbf{Z} \times \mathbf{Z}_{2}$, see below.

The proof of the following lemma is quite similar to the proof of Proposition 7 so we omit details.

Lemma 7. Let $\varepsilon$ be a YB unitary such that $\Phi_{\varphi}(\varepsilon)=\frac{1}{\sqrt{2}}$ and $\rho:=$ $\lambda_{\varepsilon} \in \operatorname{End}(M)$.

Then $\operatorname{dim}\left(\mathrm{id}, \rho^{2 n}\right)=0$ for every $n \in \mathrm{N}$.

We conclude this analysis with the following

Proposition 8. Let $\varepsilon$ and $\rho$ be as above. Then $\operatorname{dim}\left(\rho^{n}, \rho^{m}\right)=0$ for every $n \neq m$. In particular $\varepsilon$ is a braiding for $\rho$ according to the terminology of [18], Definition 4.17.

More precisely, the natural representation of the braid group $\mathrm{B}_{\infty}$ of infinite strands associated to $\varepsilon$ is a braided symmetry for $\rho$ (see [2], Section 2 and [6] for the original definitions).

Proof. $\operatorname{dim}\left(\rho^{2 h+1}, \rho^{2 k}\right)=0, h, k=0,1,2, \ldots$ since $\rho^{2 h+1}$ is direct sum of irreducibles with index 2 and $\rho^{2 k}$ is direct sum of irreducibles with index 1 .

$\operatorname{dim}\left(\rho^{2 h}, \rho^{2 k}\right)=0, h, k=0,1,2, \ldots, h \neq k$. We may assume that $h<k$. By induction $\rho^{2 h}=2^{h-1} \alpha_{1}^{h}+2^{h-1} \alpha_{1}^{h-1} \alpha_{2}$, and similarly for $k \cdot \operatorname{dim}\left(\rho^{2 h}, \rho^{2 k}\right)>0$ implies $\alpha_{1}^{k-h}=1$ or $\alpha_{1}^{k-h-1} \alpha_{2}=1$ (in $\operatorname{Sect}(M)$ ) but this is not possible by the previous result (id is not contained in $\rho^{2(k-h)}$ ).

$$
\left(\rho^{2 h+1}, \rho^{2 k+1}\right) \subset\left(\rho^{2 h+2}, \rho^{2 k+2}\right), \quad h, k=0,1, \ldots, h \neq k .
$$

The last statements follow immediately from Remark 4.

The last results contain all informations about the fusion rules relative to the ${ }^{*}$-semiring (in $\operatorname{Sect}(M)$ ) generated by $\lambda_{\varepsilon}$. A suitable comment is the following. If one thinks to $\lambda_{\varepsilon}$ as the defining representation of a quantum object in the same way as it happens for a (ordinary) compact matrix group, one can recover its "dual" taking tensors of its defining (fundamental) representation together with the conjugate and then decomposing into irreducibles.

Without assuming any supplementary structure it is interesting to recognize that the fusion algebra computed above falls within the abstract picture depicted in [12]. More precisely, our situation corresponds to the case 
$\mathrm{Z} * A_{3}$ of Theorem 3.4.11 where, however, one has to consider $r=\infty$ in formula (3.120).

Actually, an interesting problem is to check whether $\lambda_{\varepsilon}$ generates a braided tensor $C^{*}$-category of endomorphisms. A broad class of categories is classified in [12]. Our fusion rules are compatible only with the case (ii)-(b) of Theorem 8.2.11 where $r=\infty$, see p.411.

Other explicit occurrences of the fusion algebra as above seem not present in the literature. Moreover there is some evidence for them to be related to the dual object of a deformation of some group (possibly $U(2)$ ) at a primitive fourth root of unity acting on $\mathrm{O}_{2}$, cf. [23]. Such fusion rules might describe the physical spectrum of some non-rational conformal quantum theory in low-dimensional space-time.

The detailed knowledge of the monoidal $C^{*}$-category generated by $\rho \equiv \lambda_{\varepsilon}$ might thus have an important role in the study and the description of some interesting quantum symmetry like those arising in low-dimensional QFT. In this case, this quantum symmetry would be realized via (explicit) simple endomorphisms of the factor $M$. We hope to return on this point elsewhere.

\section{REFERENCES}

1. P. Akemann, private communication.

2. T. Ceccherini, S. Doplicher, C. Pinzari and J. E. Roberts, Generalization of Cuntz algebras and model actions, J. Funct. Anal. 125 (1994), 416-437.

3. R. Conti and C. Pinzari, Remarks on the index of endomorphism of Cuntz algebras, J. Funct. Anal. 142 (1996), 369-405.

4. J. Cuntz, Simple $C^{*}$-algebras generated by isometries, Comm. Math. Phys. 57 (1977), 173185.

5. J. Cuntz, Regular actions of Hopf algebras on the $C^{*}$-algebra generated by a Hilbert space, in Operator algebras, mathematical physics and low dimensional topology (R. Herman, B. Tanbay eds.), Res. Notes in Math. 5 (1993), 87-100.

6. S. Doplicher, R. Haag and J. E. Roberts, Local observables and particle statistics. I; II, Comm. Math. Phys. 23 (1971), 199-230; Comm. Math. Phys. 35 (1974), 49-85.

7. S. Doplicher and J. E. Roberts, Why there is a field algebra with a compact gauge group describing the superselection structure in particle physics, Comm. Math. Phys. 131 (1990), 51-107.

8. F. Fidaleo, and T. Isola, On the conjugate endomorphism in the infinite index case, Math. Scand. 77 (1995), 289-300.

9. F. Fidaleo and T. Isola, Minimal expectations for inclusions with atomic centres, International J. Math. 7 (1996), 307-327.

10. F. Fidaleo and T. Isola, The canonical endomorphism for infinite index inclusions, Z. Anal. Anwendungen 18 (1999), 47-66.

11. K. Fredenhagen, K.-H. Rehren and B. Schroer, Superselection sectors with braid group statistics and exchange algebras. I, Comm. Math. Phys. 125 (1989), 201-226.

12. J. Fröhlich and T. Kerler, Quantum groups, Quantum Categories and Quantum Field Theory, Lecture Notes in Math. 1542 (1993). 
13. P. de la Harpe, M. Kervaire and C. Weber, C., On the Jones polynomial, Enseign. Math. 32 (1986), 271-335.

14. F. Hiai, Minimizing Indices of Conditional Expectations onto a Subfactor, Publ. Res. Inst. Math. Sci. 24 (1988), 673-678.

15. J. Hietarinta, All solutions to the constant quantum Yang-Baxter equation in two dimensions, Phys. Lett. A 165 (1992), 245-251.

16. M. Izumi, Application of fusion rules to the classification of subfactors, Publ. Res. Inst. Math. Sci. 27 (1991), 953-994.

17. M. Izumi, Subalgebras of infinite $C^{*}$-algebras with finite Watatani index, I. Cuntz algebras, Comm. Math. Phys. 155 (1993), 157-182.

18. M. Izumi, Subalgebras of infinite $C^{*}$-algebras with finite Watatani index, II. Cuntz-Krieger algebras, Duke Math. J. 91 (1998), 409-461.

19. V. F. R. Jones, Index for subfactors, Invent. Math. 72 (1983), 1-25.

20. V. F. R. Jones, A polynomial invariant for knots via von Neumann algebras, Bull. Amer. Math. Soc. 12 (1985), 103.

21. V. F. R. Jones, Braid groups, Hecke algebras and type $\mathrm{II}_{1}$ factors, in: Geometric methods in operator algebras (Kyoto, 1983), Pitman Res. Notes in Math. Ser. 123 (1986), 242-273.

22. V. F. R. Jones, On a family of almost commuting endomorphisms, J. Funct. Anal. 122 (1994), 84-90.

23. Y. Konishi, M. Nagisa and Y. Watatani, Some remarks on actions of compact matrix quantum groups on $C^{*}$-algebras, Pacific J. Math. 153 (1992), 119-127.

24. H. Kosaki, Extension of Jones' theory of index to arbitrary subfactors, J. Funct. Anal. 66 (1986), 123-140.

25. R. Longo, Index of subfactors and statistics of quantum fields. I, Comm. Math. Phys. 126 (1989), 217-247.

26. R. Longo, Index of subfactors and statistics of quantum fields. II, Comm. Math. Phys. 130 (1990), 285-309.

27. R. Longo, Minimal index and braided subfactors, J. Funct. Anal. 109 (1992), 98-112.

28. R. Longo, A duality for Hopf algebras and for subfactors. I, Comm. Math. Phys. 159 (1994), $133-150$.

29. R. Longo and J. E. Roberts, A theory of dimension, K-Theory 11 (1997), 103-159.

30. G. Mack and V. Schomerus, Conformal field algebras with quantum symmetry from the theory of superselection sectors, Comm. Math. Phys. 134 (1990), 139-196.

31. G. Mack and V. Schomerus, Quasi Hopf quantum symmetry in quantum theory, Nucl. Phys. B370 (1992), 185-230.

32. A. Ocneanu, Quantized groups, string algebras and Galois theory for algebras, in Operator Algebras and applications, vol. II, London Math. Soc. Lecture Note Ser. 136 (1988), 119-172.

33. S. Popa, Classification of subfactors and of their endomorphisms, CBMS Regional Conf. Ser. in Math 86 (1995).

34. K.-H. Rehren, Field operators for anyons and plektons, Comm. Math. Phys. 145 (1992), 123148.

35. K.-H. Rehren, On the range of the index of subfactors, J. Funct. Anal. 134 (1995), 183-193.

36. V. Schomerus, Construction of field algebras with quantum symmetry from local observables, Comm. Math. Phys. 169 (1995), 193-236.

37. V. G. Turaev, The Yang-Baxter equation and invariants of links, Invent. Math. 92 (1988), 527-553.

38. F. Xu, New braided endomorphisms from conformal inclusions, Comm. Math. Phys. 192 (1998), 349-403.

39. Y. Watatani, Index for $C^{*}$-algebras, Mem. Amer. Math. Soc. 424 (1990).

40. H. Wenzl, Hecke algebras of type $A_{n}$ and subfactors, Invent. Math., 92 (1988), 349-383. 
41. H. Wenzl, Quantum groups and subfactors of type B, C and D, Comm. Math. Phys., 133 (1990), 383-432.

42. H. Wenzl, Representations of braid groups and the quantum Yang-Baxter equation, Pacific J. Math., 145 (1990), 153-180.

DIPARTIMENTO DI MATEMATICA

II UNIVERSITÀ DI ROMA (TOR VERGATA)

VIA DELLA RICERCA SCIENTIFICA

00133 ROMA

ITALY

Email:conti@axp.mat.uniroma2.it, fidaleo@axp.mat.uniroma2.it 